\title{
Evolutionary analysis of the short-type peptidoglycan-recognition protein gene (PGLYRP1) in primates
}

\author{
W. Liu ${ }^{1 *}$, Y.F. Yao ${ }^{1 *}$, L. Zhou ${ }^{2}$, Q.Y. Ni ${ }^{1}$ and H.L. Xu ${ }^{1,3}$ \\ ${ }^{1}$ College of Animal Science and Technology, \\ Sichuan Agricultural University, Ya’an, China \\ ${ }^{2}$ Institute of Laboratory Animals, \\ Sichuan Academy of Medical Sciences \& Sichuan Provincial People's Hospital, \\ Chengdu, China \\ ${ }^{3}$ Key Laboratory of Animal Disease and Human Health of Sichuan Province, \\ Sichuan Agricultural University, Ya’an, China \\ *These authors contributed equally to this study. \\ Corresponding author: H.L. Xu \\ E-mail: huailxu@yahoo.com
}

Genet. Mol. Res. 12 (1): 453-462 (2013)

Received July 26, 2012

Accepted November 7, 2012

Published February 8, 2013

DOI http://dx.doi.org/10.4238/2013.February. 8.10

\begin{abstract}
Short-type peptidoglycan (PGN)-recognition protein 1 (PGLYRP1), an innate immunity protein that directly breaks down the structure of microbial cell wall PGNs, plays an important role both in antibacterial defenses and several inflammatory diseases. To explore the adaptive evolution of the PGLYRPI gene in primates and provide insight into the function of this antibacterial protein, we sequenced the entire PGLYRP1 gene from Macaca thibetana and Rhinopithecus roxellana, identified the corresponding sequences from the draft genome of 8 other primates, including humans, and conducted related statistical analyses. Homology analysis showed that the identity of nucleotide and deduced amino acid sequences of PGLYRP1 among 10 primates ranged from 82.0 to $99.0 \%$ and 74.5 to $98.5 \%$, respectively. The R value (transition/
\end{abstract}


transversion) and disparity index per site also presented relatively lowbase composition biases. Selective pressure analysis for the PGLYRPI sequences among major primates revealed that both the whole gene and the substructure of $P G L Y R P 1$ are under strong purifying selection at similar levels of selective pressure among 6 major primate lineages (human, great ape, lesser ape, Old World monkey, New World monkey, and prosimian monkey). Using the Bayes empirical Bayes procedure, we also detected 2 positively selected codons (121L and $141 \mathrm{~T}$ sites) that are independent of PGN-binding and PGLYRP-specific regions, implying 2 potential key sites for the functional effect of the PGLYRP1 protein. These results demonstrated that PGLYRP1 was highly conserved at the molecular level and subjected to strong functional constraints during primate evolution.

Key words: Innate immunity; Molecular evolution; PGLYRP1 gene; Primate; Purifying selection; Positive selection

\section{INTRODUCTION}

The innate immune system, a self-defense mechanism, recognizes microorganisms through a series of pattern recognition receptors with highly conserved physiological functions (Hoffmann and Reichhart, 2002; Janeway Jr. and Medzhitov, 2002). The innate immune system of higher eukaryotes, including mammals, has several important constituent members, including CD14, Toll-like receptor 2 (Takeda and Akira, 2005), a novel family of peptidoglycan (PGN) recognition proteins (PGRPs), Nod1 and Nod2, and PGN-lytic enzymes (lysozyme and amidases) (Dziarski, 2004). Short PGRP 2 (PGLYRP1, also known as PGRP-S) - a member of the PGRP family - was cloned from several organisms from insects to mammals, including fruit fly, mosquito, silkworm, moth, cow, camel, mouse, rat, pig, and human (Yoshida et al., 1996; Kang et al., 1998; Werner et al., 2000; Rehman et al., 2001; Dimopoulos et al., 2002; Wang et al., 2003; Tydell et al., 2006). As an antibacterial protein that breaks down the structure of microbial cell wall PGNs, including Lys-type and Dap-type PGNs (Schleifer and Kandler, 1972), PGLYRP1 plays a key role in mammalian congenital self-defense against pathogenic microorganisms.

Nonhuman primates are extensively used as experimental model animals for biomedical research in fields such as transplantation studies and vaccine development against measles, Ebola, and acquired immunodeficiency syndrome owing to their high anatomical, physiological, genetic, and behavioral similarity to humans (Lackner and Veazey, 2007; Seggewiss et al., 2007). Exploration of the adaptive evolution of primates in a complex microbial environment has resulted in comprehensive analyses of coding regions for several important immune-related genes in many species. These analyses provide a wealth of information about the functional and physiological adaptation of proteins encoded by these genes. PGLYRP1, a small extracellular protein $(20 \mathrm{kDa})$ (Yoshida et al., 1996) is involved in the tertiary (gelatinase) granules of polymorphonuclear leukocytes (Dziarski, 2003), bone marrow (Dziarski, 2004), neutrophils (Tydell et al., 2002), eosinophils (Fornhem et al., 1996), corneal tissue (Ghosh et al., 2009), kidney, liver, small intestine, spleen, thymus, peripheral leukocyte, lung, and fetal spleen (Kang et al., 
1998). PGLYRP1 kills bacteria directly by activating a 2-component protein-sensing system when skin and mucous membranes contact a complicated external environment (Kashyap et al., 2011). Nevertheless, other functions such as amidase activity (Mellroth and Steiner, 2006), activation of the prophenoloxidase cascade, Toll receptor (Garver et al., 2006), and immune deficiency pathway (Kaneko et al., 2005) were also important in the environmental adaption of animals ranging from insects to mammals.

Molecular evolutionary analysis has been an important tool in the exploration of gene function through comparative genomics, in which the rate ratio $\omega$ of the number of nonsynonymous substitutions per nonsynonymous site to the number of synonymous substitutions per synonymous site has been used as an indicator of selective pressure on corresponding protein-coding genes (Shi et al., 2003). An $\omega$ value of $>1$ implies positive Darwinian selection; a value of $<1$ implies purifying selection, and a value of 1 indicates neutral selection. Although the roles of PGRPs in insects are well documented, less is known about the functions of these proteins in mammalian systems. In particular, earlier studies have rarely referred to the evolution of primate $P G L Y R P 1$. The analysis in this study is expected to provide insights into the functional significance of the gene in the course of primate evolution.

\section{MATERIAL AND METHODS}

\section{Samples}

DNA samples were isolated from the muscle tissue of Sichuan snub-nosed monkeys (Rhinopithecus roxellana) and Tibetan macaques (Macaca thibetana) at the Wildlife Protection Laboratory, Sichuan Agricultural University, Ya'an, Sichuan, China. Two PGLYRP1 gene sequences from crab-eating macaque (Macaca fascicularis) and human (Homo sapiens) were downloaded from GenBank (accession Nos. AB263124 and NM_005091, respectively). Seven additional PGLYRP1 sequences, including those of northern greater galago (Otolemur garnettii), rhesus macaque (Macaca mulatta), common marmoset (Callithrix jacchus), northern white-cheeked gibbon (Nomascus leucogenys), Sumatran orangutan (Pongo abelii), western gorilla (Gorilla gorilla), and dog (Canis familiaris) were obtained from the Ensembl Genome Database (http://www.ensembl.org).

\section{Amplifying, cloning and sequencing}

Two pairs of polymerase chain reaction (PCR) primers were designed based on the PGLYRP1 sequence of rhesus macaque using the Primer 5.0 software and synthesized by Shanghai Biological Engineering Technology Services Co. Ltd. (Shanghai, China). Nucleotide sequences of these primers were PgrpF1 (forward): 5'-CCAGCAGGGTGTGAAGCAAGAC3', PgrpR1 (reverse): 5'-ACTCACTTGTAGCCCACGTCGC-3', PgrpF2-3 (forward): 5'-AGA AGGATGCTGGGGAAGTTGTG-3', and PgrpR2-3 (reverse): 5'-GGATCTCAGGGGTCTTG GTTCAG-3'. The first pair and the latter pair were used to amplify exon 1 and exons 2-3, respectively.

PCR was performed in a thermal cycler (Mastercycler gradient, Eppendorf, Germany) with a total reaction volume of approximately $50 \mu \mathrm{L}$ containing $1 \mu \mathrm{L} 10 \mathrm{ng} / \mu \mathrm{L}$ genomic DNA, $0.5 \mu \mathrm{L}$ of each primer, $5 \mu \mathrm{L} 2 \mathrm{X}$ buffer, $25 \mu \mathrm{L} 2 \mathrm{X}$ mix, $18 \mu \mathrm{L}$ double-distilled water, and $5 \mu \mathrm{L}$ mineral oil. PCR conditions were initial denaturation at $95^{\circ} \mathrm{C}$ for $5 \mathrm{~min} ; 30$ cycles of denatur- 
ation at $95^{\circ} \mathrm{C}$ for $40 \mathrm{~s}$, annealing at $60^{\circ} \mathrm{C}$ for $45 \mathrm{~s}$ and extension at $72^{\circ} \mathrm{C}$ for $50 \mathrm{~s}$; followed by a final extension at $72^{\circ} \mathrm{C}$ for 8 min.

The amplified PGLYRP1 fragment was purified using a PCR gel extraction kit (Sangon Biotech Co., Ltd., Shanghai, China) and cloned into a pMD19-T Simple vector (TaKaRa Biotechnology Co., Ltd., Dalian, China) according to manufacturer instructions. The recombinant plasmids were sequenced directly using a Big Dye Terminator v3.1 cycle sequencing ready reaction kit (Applied Biosystems, Darmstadt, Germany) in an automatic sequencer (ABI-PRISM 3730 Genetic Analyzer, Applied Biosystems, CA, USA). The sequences were assembled in the Seqman Pro module of the Lasergene 7.1 software (DNASTAR, Madison, WI, USA) to obtain the complete coding sequence of PGLYRP1.

\section{Data analysis}

Nucleotide sequence variation was confirmed through artificial check and correction using the Chromas 1.45 (McCarthy, 1998) and SeqManII programs (DNAStar LaserGene, Madison, WI, USA). To glimpse the sequence characterization, we completed a comprehensive analysis of the sequence, including sequence homology, GC content of various codons, ratio of transition/transversion (ti/tv), disparity index, and the information of variable sites in the MegAlign program (DNAStar LaserGene) and Molecular Evolutionary Genetics Analysis 5 (Tamura et al., 2011).

The nucleotide sequence alignment was generated according to a protein sequence alignment determined previously using Molecular Evolutionary Genetics Analysis 5 (Tamura et al., 2011). Branch models with varying $\omega$ ratios among branches in the phylogeny were used to detect positive selection on particular lineages through the variable model and specify branches of interest (Yang, 2007). We conducted selective test for the overall region, structure-specific encoded regions and PGLYRP-specific fragments of the PGLYRP1 gene, respectively. The site models applied the $\omega$ ratios to various sites (among codons or amino acids in the protein) (Yang, 2007). All positions containing gaps and missing data were eliminated (Tamura et al., 2011).

\section{RESULTS}

\section{Sequence characterization of the primate PGLYRP1 gene}

We first obtained 2 nucleotide sequences of PGLYRP1 (complete coding sequence) from Tibetan macaque and Sichuan snub-nosed monkey through PCR, cloning, and sequencing (accession Nos. KC110627 and KC110628). In addition, we obtained 8 corresponding sequences from other primates from GenBank and Ensembl. In total, 10 major representative primates, including the prosimian monkey, New World monkeys, Old World monkeys, lesser apes, great apes, and humans, contained $591 \mathrm{bp}$ and coded 196 amino acids, with the exception that a 3-bp deletion occurred in the northern greater galago $(O$. garnettii). The prediction of high protein structure showed that all species have the typical features of an N-terminal signal peptide of 21 amino acids and only extracellular mature proteins. A total of 159 variable sites were detected, of which 59 were parsim-informative and 100 were singleton sites. All had 7 highly conserved cysteine residues, and 6 were paired with 3 disulfide bonds (Figure 1). 


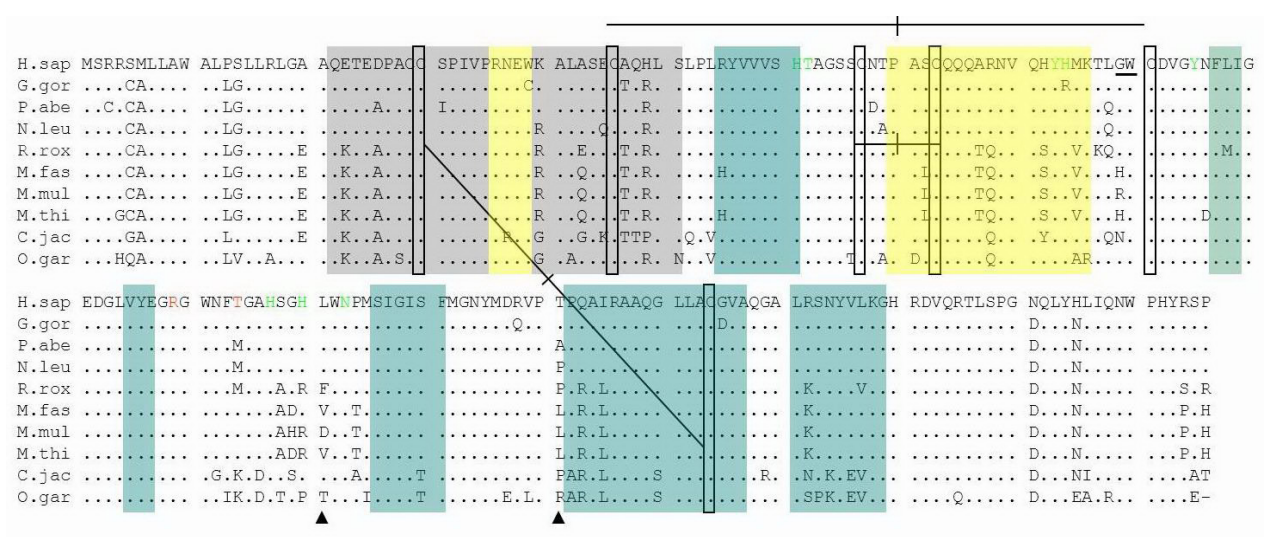

Figure 1. Alignment of the amino acid sequences of $P G R P-S$ in primates. The map was modified according to the alignment and $P G R P-S$ fine structure (Sharma et al., 2008). The signal peptide contains the first 21 amino acids. The completely conserved L-cysteine residues are in rectangles. The solid lines with a vertical bar are disulfide bond formed by L-cysteine. PGN-binding residues are indicated in green. The specific Lys-PGN recognition residues are underlined and the binding Dap-PGN are highlighted in red. Sites in blue and in gray shadows are PGN-binding sites and PGLYRP1-specific fragment, respectively, and yellow shaded sites are shared by both functional fragments. Triangles below the sequence O.gar mark positively selected sites under M8. H.sap = Homo sapiens; G.gor = Gorilla gorilla; P.abe $=$ Pongo abelii $;$ N.leu = Nomascus leucogenys $;$ R.rox $=$ Rhinopithecus roxellana $;$ M.fas = Macaca fascicularis; M.mul = Macaca mulatta $;$ M.thi = Macaca thibetana $;$ C $. j a c=$ Callithrix jacchus; O.gar = Otolemur garnettii.

We pursued the signs of sequence characterization. The ti/tv rate ratios were estimated at 1.58 under the Hasegawa et al. $(1985)$ model $(+\mathrm{G})$ with pairwise sequence comparison and joint likelihood analysis (-2356.099), indicating a low ti/tv preference. Most disparity indexes between sequences were near 0 , suggesting relatively small differences in base composition biases. The sequence homology generated by pairwise sequence alignments revealed that the identity levels of nucleotide and amino acid sequences among major primates ranged from 82.0 to $99.0 \%$ and 74.5 to $98.5 \%$, respectively (Table 1 ).

Table 1. Pairwise analysis of divergence in the PGLYRP1 sequences from major primates.

\begin{tabular}{|c|c|c|c|c|c|c|c|c|c|c|}
\hline Lineages & H. sapiens & G. gorilla & P. abelii & N. leucogenys & R. roxellana & M. mulatta & M. thibetana & M. fascicularis & C. jacchus & O. garnettii \\
\hline \multicolumn{11}{|l|}{ Human } \\
\hline Homo sapiens & - & 93.9 & 92.9 & 93.4 & 84.3 & 84.8 & 83.2 & 84.8 & 79.7 & 76.0 \\
\hline \multicolumn{11}{|l|}{ Great ape } \\
\hline Gorilla gorilla & 97.1 & - & 93.9 & 94.4 & 86.3 & 86.8 & 85.3 & 86.8 & 79.7 & 75.5 \\
\hline Pongo abelii & 96.6 & 97.1 & - & 95.9 & 87.8 & 86.3 & 84.8 & 86.3 & 80.7 & 76.5 \\
\hline \multicolumn{11}{|l|}{ Lesser ape } \\
\hline Nomascus leucogenys & 96.8 & 97.3 & 98.0 & - & 88.8 & 86.8 & 85.3 & 86.8 & 81.7 & 77.6 \\
\hline \multicolumn{11}{|l|}{ OWM } \\
\hline Rhinopithecus roxellana & 92.9 & 93.7 & 94.1 & 94.8 & - & 92.9 & 91.4 & 91.9 & 82.2 & 76.5 \\
\hline Macaca mulatta & 92.4 & 92.9 & 92.9 & 93.2 & 96.6 & - & 97.0 & 97.5 & 81.2 & 75.5 \\
\hline Macaca thibetana & 92.0 & 92.6 & 92.6 & 92.9 & 96.3 & 99.0 & - & 98.5 & 79.7 & 74.5 \\
\hline Macaca fascicularis & 92.6 & 93.1 & 93.1 & 93.4 & 96.4 & 99.2 & 99.5 & - & 81.2 & 75.0 \\
\hline \multicolumn{11}{|l|}{ NWM } \\
\hline Callithrix jacchus & 87.6 & 88.0 & 88.0 & 89.3 & 89.7 & 88.7 & 88.5 & 89.0 & - & 78.1 \\
\hline \multicolumn{11}{|l|}{ PM } \\
\hline Otolemur garnettii & 82.0 & 82.0 & 82.0 & 83.2 & 83.2 & 82.5 & 82.0 & 82.3 & 83.0 & - \\
\hline
\end{tabular}

A total of 10 PGLYRP1 sequences from major primates were subjected to pairwise analysis of sequence differences. Regions of deleted or inserted nucleotides were excluded from the calculation of divergence. The homology of amino acids is shown above diagonal and that of nucleotide sequence is below diagonal. OWM $=$ Old World monkey; NWM = New World monkey; PM = Prosimian monkey. 


\section{Selective pressure analysis of $P G L Y R P 1$ in primates}

The maximum likelihood tree reconstructed using 10 primate PGLYRP1 sequences displayed only subtle differences in misplacement between rhesus and Tibetan macaques compared with the established primate species tree (Goodman et al., 1998), suggesting that the sequences obtained are orthologous. We thus used the established species tree in all subsequent analyses (Figure 2).

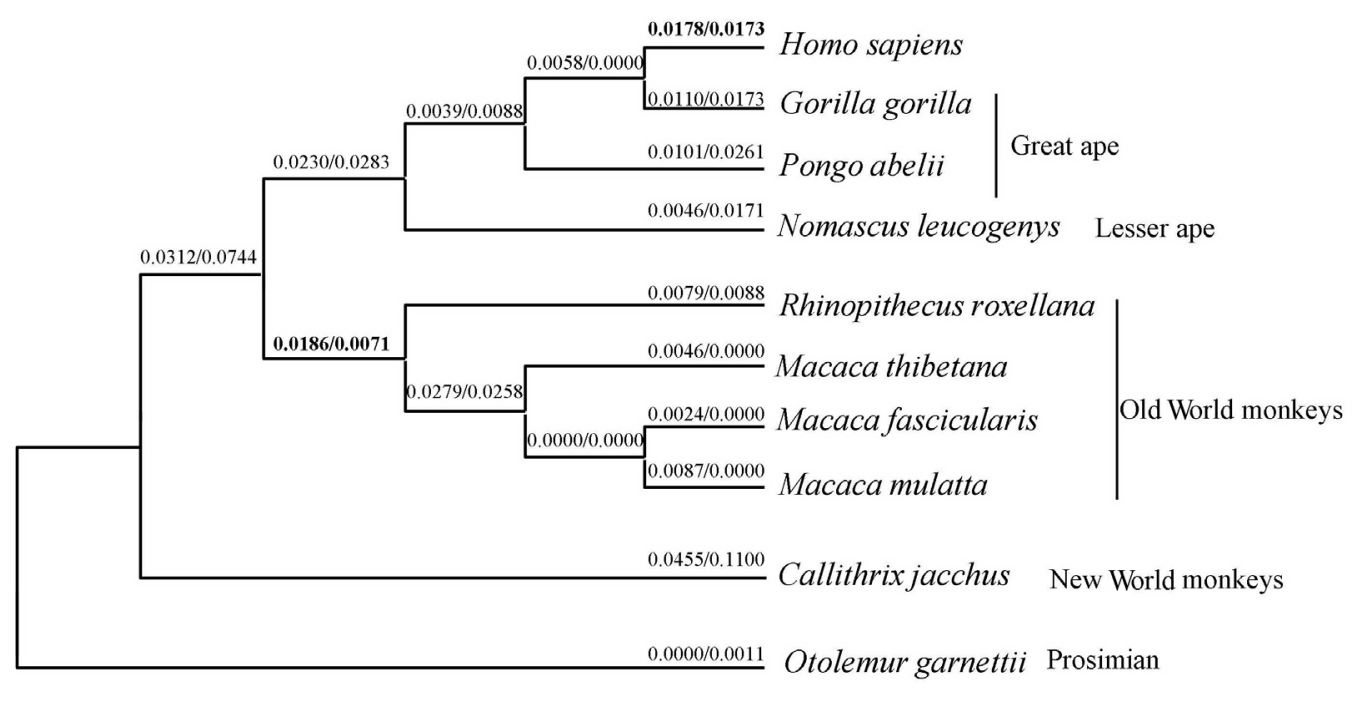

Figure 2. Evolutionary rates of PGLYRP1 in major primates. Tree topology was followed by Goodman et al. (1998). Two numbers shown along each branch correspond to maximum-likelihood estimates of the numbers of non-synonymous and synonymous substitution along that branch. For branches human and Old World monkeys, the omega values greater than 1 are in bold although omega in human branch is not significant $(\mathrm{P}>0.05)$.

To assess the selective pressure on PGLYRP1 in primates, we estimated the ratio $\omega$ of nonsynonymous to synonymous substitution rates of the genes in 10 species using a likelihood method. First, under the assumption of a uniform $\omega$ for all branches of the 10 primates (model A in Table 2), $\omega$ was estimated to be 0.4606 , which is significantly smaller than $1(\mathrm{P}<0.01$; see the comparison with model B in Table 2). This result suggests that PGLYRP1 is under an overall strong purifying selection in these primates. Second, we tested whether a model that allows different $\omega$ values for 6 major primate lineages (human, great ape, lesser ape, Old World monkey, New World monkey, and prosimian monkey; model D in Table 2) fits the data significantly better than a simpler model that disallows differences among major lineages (model C in Table 2). Model $\mathrm{D}$ did not fit the data significantly better than model $\mathrm{C}$ did $(\mathrm{P}=0.3873)$, indicating similar levels of selective pressure on PGLYRP1 among 6 major primate lineages, although $\omega$ values of $>1$ - signals of positive selection - were found in 2 major lineages: human $(\omega 1=1.0258)$ and Old World monkeys $(\omega 4=1.5862)$. Third, we examined a model in which every branch had its own $\omega($ model $\mathrm{H})$. This model was not significantly better than the uniform $\omega$ model $\mathrm{A}(\mathrm{P}=0.1454)$, suggesting that the variation of $\omega$ among 10 different species lineages is minimal. 
Table 2. Likelihood ratio test of selective pressure on PGLYRP1 in primates using branch models in PAML.

\begin{tabular}{|c|c|c|c|c|c|}
\hline Models & Parameter estimates & $\ln L$ & $\mathrm{np}$ & Models compared & $2 \Delta(\operatorname{lnL})(\mathrm{P})^{\mathrm{a}}$ \\
\hline A: All branches have the same $\omega$ & $\omega=0.4606$ & -1811.98 & 20 & \multirow{3}{*}{$\mathrm{B} v s \mathrm{~A}$} & \multirow{3}{*}{$23.08(\mathrm{P}<0.01)^{* *}$} \\
\hline B: All branches have one $\omega=1$ & $\omega=1$ & -1823.52 & 19 & & \\
\hline $\begin{array}{l}\text { C: Braches in human, great ape, lesser ape, } \\
\text { Old World monkeys, New World monkeys, and } \\
\text { prosimian have the same } \omega . \text { Other branches } \\
\text { have their own omega }\end{array}$ & $\omega=0.8147$ & -1805.28 & 21 & & \\
\hline D: Braches in human, great ape, lesser ape, & $\omega l=1.0258$ & \multirow[t]{6}{*}{-1802.66} & \multirow[t]{6}{*}{26} & \multirow[t]{6}{*}{$\mathrm{C}$ vs $\mathrm{D}$} & \multirow[t]{6}{*}{$5.24(\mathrm{P}=0.3873)$} \\
\hline Old World monkeys, New World monkeys, and & $\omega 2=0.6631$ & & & & \\
\hline prosimian monkey have $\omega 1, \omega 2, \omega 3, \omega 4, \omega 5$, & $\omega 3=0.3191$ & & & & \\
\hline and $\omega 6$, respectively. Other branches have their & $\omega 4=1.5862$ & & & & \\
\hline own omega. & $\omega 5=0.4204$ & & & & \\
\hline & $\omega 6=0.2049$ & & & & \\
\hline H: Each branch has its own $\omega$ & $\begin{array}{l}\text { Variable } \omega \text { in each } \\
\text { branch }\end{array}$ & -1800.42 & 37 & A vs $\mathrm{H}$ & $23.12(\mathrm{P}=0.1454)$ \\
\hline
\end{tabular}

$\operatorname{lnL}=$ natural logarithm of the likelihood value; $\mathrm{np}=$ number of parameters. ${ }^{\mathrm{a}}$ Twice the difference in $\operatorname{lnL}$ between the two models compared. ${ }^{*}$ Showed to be extremely significant in the statistical test.

To explore further whether some key local regions present different evolutionary patterns, we also conducted tests on the complete coding sequence for 2 subregions of the PGLYRP1 gene: the PGN-binding site region (blue and yellow shadows in Figure 1) and the PGLYRP1-specific fragment region (gray and yellow shadows in Figure 1) (Guan et al., 2005). The results indicated that the 2 subregions are also under strong purifying selection with an $\omega$ value far below $1(\omega=0.3205$ and 0.4801 , respectively; Table 3$)$.

\begin{tabular}{|c|c|c|c|c|}
\hline Structure & $\operatorname{lnL}(\omega=1)$ & $\operatorname{lnL}($ one $\omega)$ & $2 \mathrm{dL}$ & $P($ d.f. $=1)$ \\
\hline Binding region & -608.23 & $-598.95(0.2925)$ & 18.56 & $<0.01$ \\
\hline Specific fragment & -422.85 & $-419.97(0.4340)$ & 5.76 & $<0.05$ \\
\hline
\end{tabular}

$2 \mathrm{dL}=$ twice the difference in $\operatorname{lnL}$ between the two models compared; d.f. $=$ degrees of freedom.

\section{Identification of positively selected sites}

To further clarify which amino acid residues contribute to the evolutionary adaptation of PGLYRP1 in the innate immune system, six Nssites models were implemented in the CODEML program by specifying the variable Nssites $=0,1,2,3,7,8$ (and model $=0$ ). First, whether $\omega$ value among sites was variable was evaluated by comparing M0 and M3. Likelihood ratio tests (LRTs) showed that M3 fit significantly much better than M0 did (P < 0.01 ), i.e., the selective pressure was highly variable among sites. Then two pairs of models, including M1a against M2a and M7 against M8, were used to detect positive selection with LRTs. The LRTs of M1a versus M2a and M7 versus M8 were highly significant, with $2 \Delta(\operatorname{lnL})$ $=21.56$, degrees of freedom $=2, \mathrm{P}<0.01$, and $2 \Delta(\operatorname{lnL})=21.62$, degrees of freedom $=2, \mathrm{P}$ $<0.01$, respectively. The random-site models demonstrated extreme variability in selective pressure among sites and revealed the presence of a few of sites under positive selection. The positive selection sites were identified using the Bayes empirical Bayes procedure in the 
PAML software. In total, 2 codons (121L and $141 \mathrm{~T}$ sites) were significantly positively selected under model M8 (Table 4; see Figure 1). However, the 2 positively selected sites were independent of corresponding functional domains, including the PGLYRP-specific fragment and PGN-binding region.

\begin{tabular}{|c|c|c|c|c|c|}
\hline Models & Estimate of parameters & $\operatorname{lnL}$ & Models compared & $2 \Delta(\operatorname{lnL})^{a}$ & Positively selected sites \\
\hline M0 & $\omega=0.44991$ & -1779.29 & & & \\
\hline M1a & $\begin{array}{l}p 0=0.59056, p l=0.40944 \\
\omega 0=0.03404, \omega I=1.00000\end{array}$ & -1755.39 & & & Not allowed \\
\hline M2a & $\begin{array}{l}p 0=0.56819, p 1=0.38359, p 2=0.04823 \\
\omega 0=0.03202, \omega 1=1.00000, \omega 2=5.40800\end{array}$ & -1751.56 & M1a vs M2a & $\begin{array}{c}22.7 * * \\
(\mathrm{P}<0.01)\end{array}$ & None \\
\hline M3 & $\begin{array}{l}p 0=0.61632, p 1=0.35251, p 2=0.03117 \\
\omega 0=0.05387, \omega 1=1.23760, \omega 2=7.12693\end{array}$ & -1751.54 & M0 vs M3 & $\begin{array}{l}147.7 * * \\
(\mathrm{P}<0.01)\end{array}$ & $121 \mathrm{~L}^{*}$ \\
\hline M7 & $p=0.02075, q=0.02672$ & -1756.19 & & & Not allowed \\
\hline M8 & $\begin{array}{l}p 0=0.94955, p=0.04412 \\
q=0.05995(p 1=0.05045), \omega=5.31908\end{array}$ & -1751.62 & M7 vs M8 & $\begin{array}{r}23.28^{* *} \\
(\mathrm{P}<0.01)\end{array}$ & $\begin{array}{l}121 \mathrm{~L}^{* *} \\
141 \mathrm{~T}^{* *}\end{array}$ \\
\hline
\end{tabular}

$\operatorname{lnL}=$ natural logarithm of the likelihood value. ${ }^{\text {aT }}$ Twice the difference in $\ln L$ between the two models compared. The positively selected sites with $*$ and $* *$ were under 95 and 99 posterior probability values, respectively.

\section{DISCUSSION}

The physiological function of PGLYRP1 is highly conserved with respect to PGN recognition of antibacterial immunity from insects to mammals. This study is the first to survey the evolution of the short-type PGLYRP1 gene in major primate lineages. R (ti/tv) and disparity index per site of nucleotide sequences revealed low ti/tv preference and base composition biases, and a relatively high similarity of PGLYRP1 nucleotide and amino acid sequences was also found among major primates. The results showed that PGLYRP1 is highly conserved at the molecular level, which could be the result of high selective pressures necessitated based on important physiological functions against pathogens (Liu et al., 2001; Girardin and Philpott, 2004).

To test whether the conservation of PGLYRPI results from purifying selection, we calculated $\omega$ for the entire coding or local region sequences in major primates according to the maximum likelihood method (Yang, 2007). We found that PGLYRP1 genes in major primate lineages have undergone strong purifying selection, a result that we attributed to functional constraints related to the importance of PGLYRP1 in primates. The phenomenon is not unique for an intact gene and some substructures in primates (Zhang et al., 1999; Xu and Su, 2005; Wooding, 2011). Notably, when selective tests were performed among 6 major primate lineages with an independent $\omega$ value for each lineage, 6 lineages presented similar levels of selective pressure, but $\omega$ values of $>1$ were found in human and Old World monkey lineages, implying that accelerated evolution may have occurred in these 2 lineages, which means that they have undergone special adaptive evolution in the face of various pathogenic microorganisms.

The Bayes empirical Bayes method identified 121L and 141T sites under positive selection, both of which are independent of both the PGN-binding region and the PGLYRPspecific fragment according to the structure of PGLYRP1 (Guan et al., 2005). The physiological function of the PGN-binding region in PGLYRP1 is already mostly known, but the exact role of the PGLYRP-specific fragment in innate immunity remains to be fully elucidated. A previous study has shown that the PGLYRP-specific fragment might be a docking domain for 
various effectors or signaling proteins (Guan et al., 2004). Studies have examined how and to what extent differences in physiological function of the orthologous PGLYRP1 result from the 2 positive selection sites. Drosophila PGRP-SB1 and PGRP-SC1B are $N$-acetylmuramoyl L-alanine amidases, while primate PGLYRP1 is not, and human PGLYRP1 preferentially selects DAP-type PGNs (Gelius et al., 2003; Mellroth and Steiner, 2006). Hence, we believe that other PGLYRP1 fragments have played an indispensable role in the evolution of PGLYRP1 in primates. However, to help identify the amino acid changes critical for evolutionary adaptation in primates, we need additional sequences to shed light on the structure-function relationship of PGLYRP1 and the molecular underpinnings of the subregions controlling and regulating the protein function in innate immunity.

\section{ACKNOWLEDGMENTS}

Research supported by the National Natural Science Foundation of China (\#30970383), the Program for Changjiang Scholars and Innovative Research Team in Sichuan Agricultural University (\#IRT0848), and the Natural Science Foundation of Educational Commission of the Sichuan Province of China (\#08ZA076).

\section{REFERENCES}

Dimopoulos G, Christophides GK, Meister S, Schultz J, et al. (2002). Genome expression analysis of Anopheles gambiae: responses to injury, bacterial challenge, and malaria infection. Proc. Natl. Acad. Sci. U. S. A. 99: 8814-8819.

Dziarski R (2003). Recognition of bacterial peptidoglycan by the innate immune system. Cell Mol. Life Sci. 60: 1793-1804.

Dziarski R (2004). Peptidoglycan recognition proteins (PGRPs). Mol. Immunol. 40: 877-886.

Fornhem C, Peterson CG and Alving K (1996). Isolation and characterization of porcine cationic eosinophil granule proteins. Int. Arch. Allergy Immunol. 110: 132-142.

Garver LS, Wu J and Wu LP (2006). The peptidoglycan recognition protein PGRP-SC1a is essential for Toll signaling and phagocytosis of Staphylococcus aureus in Drosophila. Proc. Natl. Acad. Sci. U. S. A. 103: 660-665.

Gelius E, Persson C, Karlsson J and Steiner H (2003). A mammalian peptidoglycan recognition protein with N-acetylmuramoyl-L-alanine amidase activity. Biochem. Biophys. Res. Commun. 306: 988-994.

Ghosh A, Lee S, Dziarski R and Chakravarti S (2009). A novel antimicrobial peptidoglycan recognition protein in the cornea. Invest. Ophthalmol. Vis. Sci. 50: 4185-4191.

Girardin SE and Philpott DJ (2004). Mini-review: the role of peptidoglycan recognition in innate immunity. Eur. J. Immunol. 34: 1777-1782.

Goodman M, Porter CA, Czelusniak J, Page SL, et al. (1998). Toward a phylogenetic classification of Primates based on DNA evidence complemented by fossil evidence. Mol. Phylogenet. Evol. 9: 585-598.

Guan R, Malchiodi EL, Wang Q, Schuck P, et al. (2004). Crystal structure of the C-terminal peptidoglycan-binding domain of human peptidoglycan recognition protein Ia. J. Biol. Chem. 279: 31873-31882.

Guan R, Wang Q, Sundberg EJ and Mariuzza RA (2005). Crystal structure of human peptidoglycan recognition protein S (PGRP-S) at $1.70 \AA$ Å resolution. J. Mol. Biol. 347: 683-691.

Hasegawa M, Kishino H and Yano T (1985). Dating of the human-ape splitting by a molecular clock of mitochondrial DNA. J. Mol. Evol. 22: 160-174.

Hoffmann JA and Reichhart JM (2002). Drosophila innate immunity: an evolutionary perspective. Nat. Immunol. 3: 121-126.

Janeway CA Jr and Medzhitov R (2002). Innate immune recognition. Annu. Rev. Immunol. 20: 197-216.

Kaneko T, Golenbock D and Silverman N (2005). Peptidoglycan recognition by the Drosophila Imd pathway. J. Endotoxin. Res. 11: 383-389.

Kang D, Liu G, Lundstrom A, Gelius E, et al. (1998). A peptidoglycan recognition protein in innate immunity conserved from insects to humans. Proc. Natl. Acad. Sci. U. S. A. 95: 10078-10082.

Kashyap DR, Wang M, Liu LH, Boons GJ, et al. (2011). Peptidoglycan recognition proteins kill bacteria by activating protein-sensing two-component systems. Nat. Med. 17: 676-683.

Lackner AA and Veazey RS (2007). Current concepts in AIDS pathogenesis: insights from the SIV/macaque model. Annu. 
Rev. Med. 58: 461-476.

Liu C, Xu Z, Gupta D and Dziarski R (2001). Peptidoglycan recognition proteins: a novel family of four human innate immunity pattern recognition molecules. J. Biol. Chem. 276: 34686-34694.

McCarthy C (1998). Chromas 1.45. School of Health Science. Griffith University, Southport, Queensland.

Mellroth P and Steiner H (2006). PGRP-SB1: an N-acetylmuramoyl L-alanine amidase with antibacterial activity. Biochem. Biophys. Res. Commun. 350: 994-999.

Rehman A, Taishi P, Fang J, Majde JA, et al. (2001). The cloning of a rat peptidoglycan recognition protein (PGRP) and its induction in brain by sleep deprivation. Cytokine 13: 8-17.

Schleifer KH and Kandler O (1972). Peptidoglycan types of bacterial cell walls and their taxonomic implications. Bacteriol. Rev. 36: 407-477.

Seggewiss R, Lore K, Guenaga FJ, Pittaluga S, et al. (2007). Keratinocyte growth factor augments immune reconstitution after autologous hematopoietic progenitor cell transplantation in rhesus macaques. Blood 110: 441-449.

Sharma P, Singh N, Sinha M, Sharma S, et al. (2008). Crystal structure of the peptidoglycan recognition protein at $1.8 \AA$ resolution reveals dual strategy to combat infection through two independent functional homodimers. J. Mol. Biol. 378: 923-932.

Shi J, Xi H, Wang Y, Zhang C, et al. (2003). Divergence of the genes on human chromosome 21 between human and other hominoids and variation of substitution rates among transcription units. Proc. Natl. Acad. Sci. U. S. A. 100: 8331-8336.

Takeda K and Akira S (2005). Toll-like receptors in innate immunity. Int. Immunol. 17: 1-14.

Tamura K, Peterson D, Peterson N, Stecher G, et al. (2011). MEGA5: molecular evolutionary genetics analysis using maximum likelihood, evolutionary distance, and maximum parsimony methods. Mol. Biol. Evol. 28: 2731-2739.

Tydell CC, Yount N, Tran D, Yuan J, et al. (2002). Isolation, characterization, and antimicrobial properties of bovine oligosaccharide-binding protein. A microbicidal granule protein of eosinophils and neutrophils. J. Biol. Chem. 277: 19658-19664.

Tydell CC, Yuan J, Tran P and Selsted ME (2006). Bovine peptidoglycan recognition protein-S: antimicrobial activity, localization, secretion, and binding properties. J. Immunol. 176: 1154-1162.

Wang ZM, Li X, Cocklin RR, Wang M, et al. (2003). Human peptidoglycan recognition protein-L is an N-acetylmuramoylL-alanine amidase. J. Biol. Chem. 278: 49044-49052.

Werner T, Liu G, Kang D, Ekengren S, et al. (2000). A family of peptidoglycan recognition proteins in the fruit fly Drosophila melanogaster. Proc. Natl. Acad. Sci. U. S. A. 97: 13772-13777.

Wooding S (2011). Signatures of natural selection in a primate bitter taste receptor. J. Mol. Evol. 73: 257-265.

$\mathrm{Xu} \mathrm{HL}$ and Su B (2005). Genetic evidence of a strong functional constraint of neurotrypsin during primate evolution. Cytogenet. Genome Res. 108: 303-309.

Yang Z (2007). PAML 4: phylogenetic analysis by maximum likelihood. Mol. Biol. Evol. 24: 1586-1591.

Yoshida H, Kinoshita K and Ashida M (1996). Purification of a peptidoglycan recognition protein from hemolymph of the silkworm, Bombyx mori. J. Biol. Chem. 271: 13854-13860.

Zhang YW, Ryder OA and Zhang YP (1999). Sequence evolution of the CCR5 chemokine receptor gene in primates. Mol. Biol. Evol. 16: 1145-1154. 